\title{
Ultimate fast optical switching of a planar microcavity in the telecom wavelength range
}

\author{
Georgios Ctistis, ${ }^{1, a)}$ Emre Yuce, ${ }^{1,2}$ Alex Hartsuiker, ${ }^{1,2}$ Julien Claudon, ${ }^{3}$ Maela Bazin, ${ }^{3}$ \\ Jean-Michel Gérard, ${ }^{3}$ and Willem L. Vos ${ }^{1, b)}$ \\ ${ }^{1}$ Complex Photonic Systems (COPS), MESA + Institute for Nanotechnology, University of Twente, \\ 7500 AE Enschede, The Netherlands \\ ${ }^{2}$ Center for Nanophotonics, FOM Institute for Atomic and Molecular Physics (AMOLF), \\ 1098 XG Amsterdam, The Netherlands \\ ${ }^{3}$ CEA-CNRS-UJF "Nanophysics and Semiconductors" Joint Laboratory, CEA/INAC/SP2M, \\ 38054 Grenoble Cedex 9, France
}

(Received 16 February 2011; accepted 31 March 2011; published online 22 April 2011)

\begin{abstract}
We have studied a GaAs-AlAs planar microcavity with a resonance near $1300 \mathrm{~nm}$ in the telecom range by ultrafast pump-probe reflectivity. By the judicious choice of pump frequency, we observe an ultimate fast and reversible decrease in the resonance frequency by more than half a linewidth due to the instantaneous electronic Kerr effect. The switch-on and switch-off of the cavity is only limited by the cavity storage time of $\tau_{\mathrm{cav}}=0.3 \mathrm{ps}$ and not by intrinsic material parameters. Our results pave the way to supraterahertz switching rates for on-chip data modulation and real-time cavity quantum electrodynamics. (C) 2011 American Institute of Physics. [doi:10.1063/1.3580615]
\end{abstract}

Switches are widely applied and necessary ingredients in modulation and computing schemes. ${ }^{1}$ The recent progress on photonic integrated circuits ${ }^{2,3}$ promises to overtake boundaries set by conventional switching technology. To do so, ultrafast switching of photonic cavities is crucial as it allows the capture or release on demand of photons, ${ }^{4-6}$ which is relevant to on-chip communication with light as information carrier, ${ }^{7}$ and to high-speed miniature lasers. ${ }^{8}$ Ultrafast switching would also permit the quantum electrodynamical manipulation of coupled cavity-emitter systems ${ }^{9}$ in real time. Switching the optical properties of photonic nanostructures is achieved by changing the refractive index of the constituent materials. To date, however, the switching speed has been limited by material properties ${ }^{10-13}$ but not by optical considerations. To achieve ultimate fast switching of a cavity two challenges arise. First, both the switch-on and switch-off times $\tau_{\text {on }}$ and $\tau_{\text {off }}$ must be shorter than all other relevant time scales for the system, i.e., the cavity storage time in photon capture/release experiments $\tau_{\text {cav }}$, or the vacuum Rabi period $\tau_{\text {Rabi }}$ for a strongly coupled emittercavity system. ${ }^{14}$ Second, the refractive index change must be large enough to switch the cavity resonance by at least half a linewidth.

Here, we demonstrate the ultimate fast switching of the resonance of a planar cavity in the well-known GaAs/AlAs system in the telecom wavelength range. We exploit the instantaneously fast electronic Kerr effect by the judicious tuning of the pump and probe frequencies relative to the semiconductor band gap. We observe that the speed of the switching is then only limited by the dynamics of the light in our cavity $\left(\tau_{\text {cav }}=0.3 \mathrm{ps}\right)$, but not by the intrinsic material parameters.

Instantaneous on- and off-switching with vanishing $\tau_{\text {on }}$ and $\tau_{\text {off }}$ is feasible with the well-known nonlinear refractive index from nonlinear optics. ${ }^{15}$ Physically the electronic Kerr

\footnotetext{
a) Electronic mail: g.ctistis@utwente.nl.

${ }^{b)}$ Electronic mail: W.L.Vos@tnw.utwente.nl.
}

effect is the fastest Kerr phenomenon on account of the small electron mass. In many practical situations, however, nondegenerate two-photon absorption overwhelms any instantaneous effect and therefore also the dispersive electronic Kerr effect. $^{12,15}$ In order to avoid two-photon absorption and to access the electronic Kerr switching regime, we designed our experiment to operate with low energy pump photons, see Fig. 1(a). First of all, the energy of the pump photons is chosen below half the semiconductor band gap energy $\left(E_{\text {pump }}<1 / 2 E_{\text {gap }}\right)$. Second, the energies of the pump and probe photons are chosen so that their sum does not exceed the band gap energy of the semiconductor $\left(E_{\text {probe }}+E_{\text {pump }}\right.$ $\left.\leq E_{\text {gap }}\right){ }^{16}$ These conditions serve to suppress instantaneous two-photon generation of free carriers and to perform electronic Kerr switching at elevated frequencies ( $E_{\text {probe }}$ $\left.>1 / 2 E_{\text {gap }}\right)$, including the telecom band, with a broad range of semiconductors.

We have performed our experiments on a planar microcavity grown by means of molecular-beam epitaxy. The sample consists of a $\lambda$-thick GaAs layer $(d=376 \mathrm{~nm})$ sandwiched between two Bragg stacks consisting of 7 and 19 pairs of $\lambda / 4$ thick layers of nominally pure GaAs $\left(\mathrm{d}_{\mathrm{GaAs}}\right.$ (a)

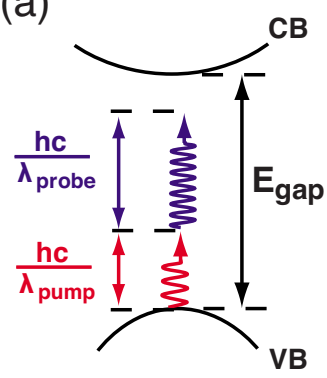

(b)

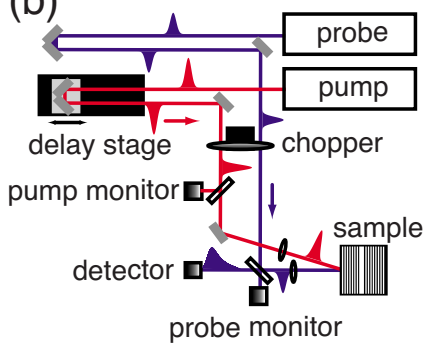

FIG. 1. (Color online) (a) Schematic energy diagram. The frequency of the probe beam is resonant with the cavity. The pump frequency is tuned such that the sum of pump and probe energies are less than the energy gap of GaAs to avoid two-photon absorption. (b) Schematic of the setup. The probe-beam path is shown in dark, the pump-beam path in light color. 


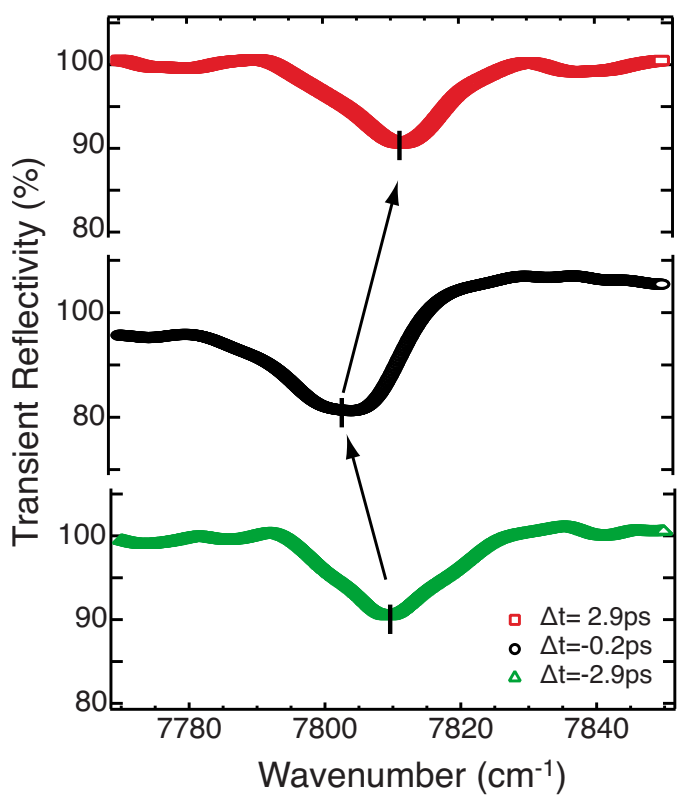

FIG. 2. (Color online) Transient reflectivity vs frequency spectra for three different delay times, $\Delta t=-2.9 \mathrm{ps}$ (triangles), $\Delta t=-0.2 \mathrm{ps}$ (circles), and $\Delta t=+2.9 \mathrm{ps}$ (squares). We observe an ultrafast shift in the cavity resonance (ticks) to lower frequencies and back to its original frequency.

$=94 \mathrm{~nm})$ or AlAs $\left(\mathrm{d}_{\mathrm{AlAs}}=110 \mathrm{~nm}\right)$. The cavity was designed such that the resonance occurs at $\lambda_{0}=1280 \mathrm{~nm}$ in the Original $(\mathrm{O})$ telecom band. Measuring the linewidth of the cavity resonance we obtained a quality factor $\mathrm{Q}=320 \pm 30$ corresponding to a cavity storage time of $\tau_{\text {cav }}=0.3 \mathrm{ps}$.

To perform Kerr-switching on a semiconductor microcavity, we have built a set-up illustrated in Fig. 1(b), consisting of two independently tunable optical parametric amplifiers (OPA, Topas) that are the sources of the pump and probe beams. The pump beam can be tuned down to $4200 \mathrm{~cm}^{-1}$ $(2400 \mathrm{~nm})$ and the probe beam is tuned to cavity resonance at $7810 \mathrm{~cm}^{-1}(1280 \mathrm{~nm})$. Under these conditions, we solely pump GaAs (the refractive index change in AlAs is much smaller and can be neglected here). The pump beam has a larger Gaussian focus $(75 \mu \mathrm{m}$ full width at half maximum) than the probe beam $(28 \mu \mathrm{m})$, to ensure that only the central flat part of the pump focus is probed and that the probed region is homogeneously pumped. The OPAs have pulse durations $\tau_{\mathrm{P}}=0.12 \pm 0.01 \mathrm{ps}$. The delay $\Delta t$ between pump and probe pulse is set by a delay stage with a resolution of 0.01 ps. A versatile measurement scheme was developed to compensate for possible pulse-to-pulse variations in the output of our laser. ${ }^{17}$

Figure 2 shows transient reflectivity versus frequency spectra for three different pump-probe delay times. One can see that the cavity resonance redshifts when approaching pump-probe overlap. Afterwards, at positive delay times, the cavity resonance blueshifts back to its original frequency. By modeling the transient reflectivity trough with a Lorentzian, both the resonance frequency $\omega_{0}$ and the width of the resonance $\Gamma$ are obtained for every time delay $\Delta t$. The unswitched cavity resonance frequency $\left(\omega_{0}=7810 \mathrm{~cm}^{-1}\right)$ and width $\left(\Gamma=12 \mathrm{~cm}^{-1}\right)$ are obtained at a delay of $\Delta t=-5 \mathrm{ps}$, confirming a cavity quality factor of $\mathrm{Q}=320$.

Figure 3(a) shows the switching of the cavity resonance due to the electronic Kerr effect. The resonance frequency as a function of delay near pump and probe coincidence is ob-

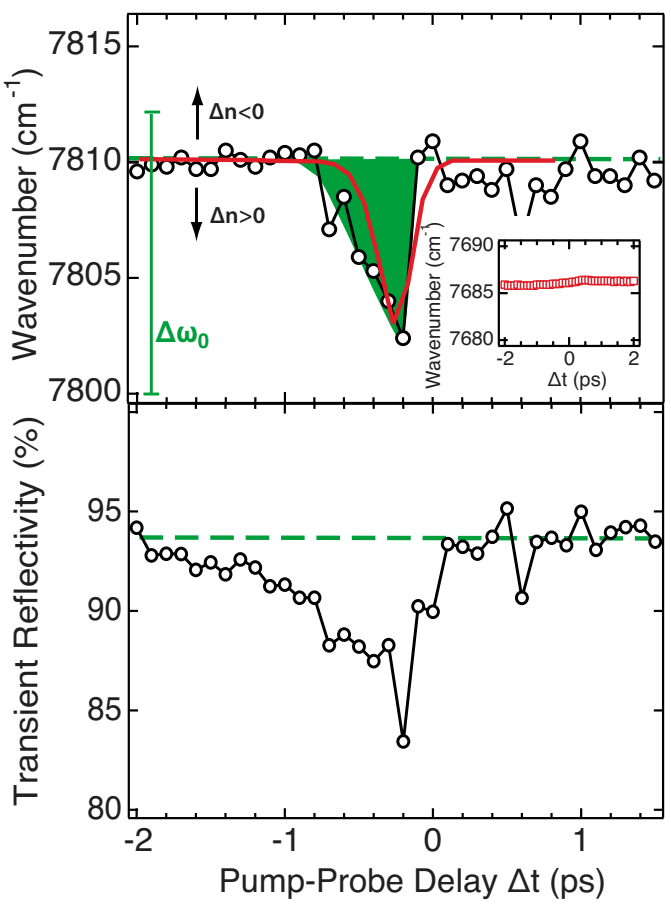

FIG. 3. (Color online) (Upper panel) Measured (open symbols) and modeled (line) resonance frequency vs pump-probe delay. The pump frequency is at $4166 \mathrm{~cm}^{-1}$. The resonance frequency reaches its maximum shift at $-0.2 \mathrm{ps}$ and quickly returns to its unswitched value. The model matches the experiment well. The inset shows no switch when the pump frequency is at $5000 \mathrm{~cm}^{-1}$ for comparison. (Lower panel) Transient reflectivity vs pumpprobe delay at the cold cavity resonance frequency. The transient reflectivity shows similar reversible behavior as the resonance.

tained from spectra similar to those shown in Fig. 2. The dashed horizontal line denotes the unswitched resonance frequency $\omega_{0}$. The measured resonance frequency shifts to a lower frequency starting at $-0.8 \mathrm{ps}$ and reaching its maximum shift at $-0.2 \mathrm{ps}$. Subsequently, the resonance frequency shifts back to its original frequency $\omega_{0}$. The dynamic frequency shift $\Delta \omega=7 \mathrm{~cm}^{-1}$ clearly exceeds one half of the cold cavity linewidth. We conclude from the shift to a lower frequency that the refractive index is increased by $0.1 \%$, which corresponds to a positive Kerr coefficient for GaAs. ${ }^{16}$ To confirm our interpretation we performed calculations with a dynamic Fabry-Pérot model, ${ }^{18,19}$ taking solely into account the refractive index change of GaAs due to the electronic Kerr effect. We observe an excellent quantitative agreement between the measured and calculated shifts for the amplitude and the temporal evolution. Our results furthermore demonstrate that our method serves to truly inhibit excitation of free carriers since there is no blueshift of the resonance at $\Delta t>0$ ps. For comparison, the inset shows the result when the pump frequency is increased to $5000 \mathrm{~cm}^{-1}$ in the free carrier regime. There is no instantaneous shift in the resonance in the given range as expected; the free-carrier excitation leads to a blueshift after a time of 3 ps. ${ }^{12}$ The very good agreement between the calculation and our experimental results firmly confirm the ultimate fast electronic Kerr switching of our photonic microcavity.

Figure 3(b) shows the transient reflectivity of the unswitched resonance frequency as a function of pump-probe delay. The relatively high reflectivity of the trough $\left(R_{\text {trough }}\right.$ $=90 \%$ ) is a result of the asymmetric cavity design. One can see that the transient reflectivity quickly changes from high 
to low (at pump-probe overlap) and back to high, within 1.5 ps. This decrease is a result of the change in the refractive index of GaAs not only in the cavity but also in the mirrors, which leads to a higher contrast in the Bragg stack.

The shape of the resonance shift in Fig. 3(a) is strikingly asymmetric. The asymmetry is a direct result of the light dynamics in the cavity. In our experiment we are probing the change in the dielectric function $\Delta \varepsilon$ of the material induced by $\mathbf{P}^{(3)}{ }^{15}$ The magnitude of the observed frequency shift $\Delta \omega$ is given by the overlap integral of the pump and probe, the latter has been stored in the cavity and subsequently escaped, at a certain delay time $\Delta t$,

$$
\frac{\Delta \omega(\Delta t)}{\omega_{0}(0)}=\frac{\Delta \varepsilon(\Delta t)}{\varepsilon(0)}=\frac{\chi^{(3)}}{\varepsilon(0)} \int E\left(\omega_{0}, t^{\prime}-\Delta t\right) E\left(\omega_{2}, t^{\prime}\right) d t^{\prime} .
$$

Hence, we tomographically sample the probe with the shorter pump. Thus, while scanning $\Delta t$, we obtain a shift that follows the cavity envelope. The shift is maximal at $-0.2 \mathrm{ps}$ when the cavity field is also maximum.

We have demonstrated for the first time the switching of a semiconductor microcavity at telecom wavelengths using the electronic Kerr effect. Our system also serves as a model system, since the nature of the switch process can be employed in any realization of a semiconductor cavity. We observed that the switching speed is limited by the cavity storage time and not by material properties. The 0.3 ps storage time in our work paves the way to subpicosecond real-time data modulation. Since switching using the electronic Kerr effect is repeatable, on-chip supraterahertz switching rates are feasible. Our results also open an avenue toward ultrafast control of all solid-state cavity quantum electrodynamical systems that exploit the strong coupling regime of quantum wells or quantum dots in optical microcavities. ${ }^{14}$
We thank Allard Mosk, Harm Dorren, Huib Bakker, and Pepijn Pinkse for stimulating discussions. This research was supported by Smartmix Memphis, NWO-Vici (to W.L.V.), and the QSWITCH ANR project (to J.M.G.). This work is also part of the research program of FOM, which is financially supported by NWO.

${ }^{1}$ S. M. Sze, Semiconductor Devices: Physics and Technology (Wiley, New York, 1985).

${ }^{2}$ S. Noda and T. Baba, Roadmap on Photonic Crystals (Kluwer, Boston, 2003).

${ }^{3}$ S. Assefa, F. Xia, and Y. A. Vlasov, Nature (London) 464, 80 (2010).

${ }^{4}$ P. M. Johnson, A. F. Koenderink, and W. L. Vos, Phys. Rev. B 66, 081102 (2002).

${ }^{5}$ V. R. Almeida, C. A. Barrios, R. R. Panepucci, and M. Lipson, Nature (London) 431, 1081 (2004).

${ }^{6}$ T. Tanabe, M. Notomi, E. Kuramochi, A. Shinya, and H. Taniyama, Nat. Photonics 1, 49 (2007).

${ }^{7}$ M. F. Yanik and S. Fan, Phys. Rev. Lett. 92, 083901 (2004).

${ }^{8}$ S. G. Hense and M. Wegener, Phys. Rev. B 55, 9255 (1997).

${ }^{9}$ J.-M. Gérard, Top. Appl. Phys. 90, 269 (2003).

${ }^{10}$ D. A. Mazurenko, R. Kerst, J. I. Dijkhuis, D. A. Kurdyukov, and A. B. Pevtsov, Appl. Phys. Lett. 86, 041114 (2005).

${ }^{11}$ S. W. Leonard, H. M. van Driel, J. Schilling, and R. B. Wehrspohn, Phys. Rev. B 66, 161102 (2002).

${ }^{12}$ P. J. Harding, T. G. Euser, Y. R. Nowicki-Bringuier, J.-M. Gérard, and W. L. Vos, Appl. Phys. Lett. 91, 111103 (2007)

${ }^{13}$ X. Hu, P. Jiang, C. Ding, H. Yang, and Q. Gong, Nat. Photonics 2, 185 (2008)

${ }^{14}$ G. Khitrova, H. M. Gibbs, M. Kira, S. W. Koch, and A. Scherer, Nat. Phys. 2, 81 (2006).

${ }^{15}$ R. W. Boyd, Nonlinear Optics (Academic, San Diego, 1992).

${ }^{16}$ A. Hartsuiker, P. J. Harding, Y. Nowicki-Bringuier, J. M. Gérard, and W. L. Vos, J. Appl. Phys. 104, 083105 (2008); P. J. Harding, T. G. Euser, and W. L. Vos, J. Opt. Soc. Am. B 26, 610 (2009).

${ }^{17}$ T. G. Euser, P. J. Harding, and W. L. Vos, Rev. Sci. Instrum. 80, 073104 (2009).

${ }^{18}$ S. Marzenell, R. Beigang, and R. Wallenstein, Appl. Phys. B: Lasers Opt. 71, 185 (2000).

${ }^{19}$ M. Born and E. Wolf, Principles of Optics, 7th ed. (Cambridge University Press, Cambridge, 2002). 


\title{
Addendum: "Ultimate fast optical switching of a planar microcavity in the telecom wavelength range" [Appl. Phys. Lett. 98, 161114 (2011)]
}

\author{
Georgios Ctistis, ${ }^{1, a)}$ Emre Yuce, ${ }^{1,2}$ Alex Hartsuiker, ${ }^{1,2}$ Julien Claudon, ${ }^{3}$ Maela Bazin, ${ }^{3}$ \\ Jean-Michel Gérard, ${ }^{3}$ and Willem L. Vos ${ }^{1}$ \\ ${ }^{1}$ Complex Photonic Systems (COPS), MESA+Institute for Nanotechnology, University of Twente, \\ 7500 AE Enschede, The Netherlands \\ ${ }^{2}$ Center for Nanophotonics, FOM Institute for Atomic and Molecular Physics (AMOLF), 1098 XG Amsterdam, \\ The Netherlands \\ ${ }^{3}$ CEA-CNRS-UJF "Nanophysics and Semiconductors" Joint Laboratory, CEA/INAC/SP2M, \\ 38054 Grenoble Cedex 9, France
}

(Received 18 October 2011; accepted 22 October 2011; published online 7 November 2011)

[doi:10.1063/1.3660223]

We have recently reported on the ultimate fast optical switching of semiconductor micro cavities by means of the electronic Kerr effect. ${ }^{1}$ We demonstrated that the switching of the cavity resonance takes place within $\tau_{c a v}=0.3 \mathrm{ps}$ and that the switching time is only limited by the quality factor $Q$ of the cavity.

It was brought to our attention that we had not specified the pump and probe intensities used in our experiments. Since these parameters are crucial for the understanding of the underlying non-linear optical processes, we report them here. First of all, we used a low probe beam intensity to avoid non-linear effects caused by the probe beam during the measurements. We, therefore, set the probe beam intensity to $I_{p r}=0.18 \mathrm{pJ} /(\mu \mathrm{m})^{2}$ at $\lambda=1300 \mathrm{~nm}$, which relates to an intracavity intensity of $I_{p r, c a v}=9 \mathrm{pJ} /(\mu \mathrm{m})^{2}$, due to the enhancement caused by the cavity resonance. ${ }^{2}$

The pump beam intensity is one order of magnitude stronger. Its precise value results from a trade-off between the optimization of the electronic Kerr effect and the minimization of free-carrier excitation. We, therefore, chose the pump intensity to a value, which can also be employed in cavities with dimensions of a few microns, namely, an intensity of $I_{p u}=70 \mathrm{pJ} /(\mu \mathrm{m})^{2}$ at $\lambda=2400 \mathrm{~nm}$. A detailed study of the intensities will be performed in the future.

${ }^{1}$ G. Ctistis, E. Yüce, A. Hartsuiker, J. Claudon, M. Bazin, J.-M. Gérard, and W. L. Vos, Appl. Phys. Lett. 98, 161114 (2011).

${ }^{2}$ A. E. Siegman, Lasers (University Science Books, Mill Valley, CA, 1986).

a)Electronic mail: g.ctistis@utwente.nl. 